\title{
Perspective
}

PERSPECTIVE Actualité en histoire de l'art

4 | 2006

La monographie d'artiste

\section{Gentile da Fabriano. Espaces ouverts à l'interprétation}

\section{Mauro Minardi}

\section{(2) OpenEdition}

1 Journals

\section{Édition électronique}

URL : http://journals.openedition.org/perspective/4222

DOI : $10.4000 /$ perspective.4222

ISSN : 2269-7721

\section{Éditeur}

Institut national d'histoire de l'art

\section{Édition imprimée}

Date de publication : 31 décembre 2006

Pagination : 614-620

ISSN : 1777-7852

\section{Référence électronique}

Mauro Minardi, «Gentile da Fabriano. Espaces ouverts à l'interprétation », Perspective [En ligne], 4 |

2006, mis en ligne le 31 mars 2018, consulté le 01 octobre 2020. URL : http://

journals.openedition.org/perspective/4222 ; DOI : https://doi.org/10.4000/perspective.4222 


\section{Gentile da Fabriano. Espaces ouverts à l'interprétation}

\section{Mauro Minardi}

Une monographie classique sur Gentile da Fabriano c'est-à-dire fondée sur un excursus de l'activité du peintre, un catalogue raisonné de ses œuvres et une documentation annexe " ne serait pas nécessaire aujourd'hui ${ }^{1}$. C'est ainsi que débute le dernier ouvrage monographique important écrit sur le maître en 1992 et cette même affirmation pourrait à nouveau être prononcée aujourd'hui et s'étendre à d'autres personnalités de l'art italien du Quattrocento. Non parce que les monographies existantes ont fourni des réponses définitives aux nombreuses questions encore ouvertes que posent l'activité et la portée de l'œuvre de Gentile da Fabriano, mais parce que les aspects de la recherche les plus stimulants concernant ce maître sont à l'évidence les analyses ponctuelles sur des œuvres et des contextes singuliers ou des approches méthodologiques spécifiques.

En mettant en jeu différentes expériences et postures scientifiques qui vont du connoisseurship aux études sur le collectionnisme jusqu'aux importantes recherches techniques et archivistiques, les volumes édités en 2005-2006 à l'occasion de la première exposition consacrée à l'artiste des Marches et aux restaurations récentes des chefs-d'œuvre florentins ont réuni matériels et problématiques qu'un seul chercheur ayant l'intention d'écrire un livre sur l'œuvre complète de Gentile da Fabriano aurait rassemblés avec beaucoup de difficulté. Mais si pour le moment une nouvelle monographie sur ce peintre ne s'impose pas, c'est aussi parce que parmi celles que nous connaissons, certaines sont le fruit de recherches rigoureusement menées.

Mais à quel moment de l'historiographie des $\mathrm{XIX}^{\mathrm{e}}$ et $\mathrm{XX}^{\mathrm{e}}$ siècles peut-on véritablement parler d'une monographie sur le maître de Fabriano ? Selon les paramètres que l'on donne aujourd'hui à ce genre, sûrement à partir du brillant texte de Luigi Grassi datant de $1953^{2}$. À l'aide de précisions stylistiques d'une grande finesse, le choix original des cadrages photographiques pour les planches en noir et blanc, la sélection rigoureuse du catalogue éliminant de mauvaises attributions, cette monographie miniature constitue l'important avant-propos des publications successives de Keith Christiansen (1982) et Andrea De Marchi (1992) qui, dans l'optique d'une telle approche, demeurent les références majeures auxquelles se reportent les chercheurs aujourd'hui encore ${ }^{3}$. En partant de méthodes déterminées, il est possible d'établir un rapport dialectique avec les études ponctuelles de façon à sonder les éléments originaux ayant émergé au cours des dernières décennies et en particulier le catalogue de l'exposition de Fabriano consacrée au peintre en 2006.

\section{Biographie et sources documentaires}

Concernant la biographie du maître et donc les sources documentaires à partir desquelles s'est construit son itinéraire artistique, ni les monographies du $\mathrm{Xx}^{\mathrm{e}}$ siècle ni les recherches conduites en vue de la préparation de l'exposition n'ont apporté de nouveautés importantes. Pour cette raison, nous devons essentiellement nous reporter aux dépouillements d'archives réalisés au cours du XIX ${ }^{\mathrm{e}}$ siècle, brillamment recueillis et mis à jour en 1896 par Adolfo Venturi dans un commentaire exemplaire du bref texte rédigé par Vasari dans les Vite sur ce peintre dont les éléments biographiques croisent ceux de son élève Pisanello ${ }^{4}$. Ce livre proposait un appareil documentaire précis et rigoureux pour l'époque, auquel s'ajoutait un catalogue des œuvres autographes et de celles faussement attribuées et perdues, dans lequel l'expérience du connaisseur avait partie prenante. À cet aperçu furent ensuite intégrées différentes sourcesdocumentaires ${ }^{5}$ jusqu'àl'importantedécouverte relative au cycle de Foligno dont nous parlerons plus loin. Malgré cela, nous sommes encore, pour certains aspects, redevables des informations fournies au milieu du Xv siècle par Bartolomeo Facio et un siècle plus tard par Vasari. Le premier, qui avait connu Pisanello, élève de Gentile à Venise, à la cour napolitaine d'Alphonse d'Aragon, est le seul à mentionner le sujet de la peinture murale réalisée à Sienne en 1425, une Madone à l'enfant entourée par quatre saints, ainsi qu'à identifier d'autres thèmes dans des fresques vénitiennes aujourd'hui perdues, certains épisodes du conflit opposant Frédéric Barberousse et le pape Alexandre III au Palais ducal et un tourbillon de vent dévastateur ( "turbinem") ailleurs dans cette même ville ${ }^{6}$. Facio mentionne également l'existence d'un portrait disparu du pape Martin V et de dix cardinaux 
(pour peu qu'il s'agisse vraiment d'une œuvre de Gentile). Ce qui compte dans cette biographie presque contemporaine du peintre est l'emphase de l'incipit lorsqu'il est question de l'activité de fresquiste du maître, réduite aujourd'hui à quelques exemples ayant survécu aux destructions. L'humaniste exaltait ces entreprises décoratives en les présentant presque comme le pivot du parcours de Gentile, notamment les travaux réalisés à Venise et ceux de la basilique de Saint-Jean-deLatran à Rome. Lorsque, au milieu du XvI siècle, Vasari rédige le portrait du peintre, il part précisément de cette expérience romaine pour construire son éloge auquel il ajoute la mention d'une fresque, inconnue aujourd'hui, réalisée par Gentile au-dessus de la sépulture du cardinal Alamanno Adimari à Sainte-Marie-Nouvelle à Rome, dont il précise le thème et rappelle l'admiration qu'elle suscita chez Michel-Ange ${ }^{7}$.

\section{Documents et lecture stylistique}

Devant les découvertes documentaires récentes concernant la peinture des Marches du début de la Renaissance (qui ont en partie renversé les identités biographiques réputées immuables), le caractère laconique des rares trouvailles relatives à Gentile surprend. Nous savons certes désormais que ses parents se sont mariés vers 1375 ou peu de temps avant, mais cette donnée ne nous permet cependant pas d'avancer de certitudes quant à la date de naissance exacte du maître ${ }^{8}$. De même que cette nouveauté naît des recherches menées à Fabriano, une autre révélation, plus importante cette fois, a surgi d'enquêtes conduites à Foligno concernant des paiements reçus par Gentile et quelques-uns de ses collaborateurs, provenant de différentes régions, entre 1411 et 1412, pour des fresques exécutées dans la demeure des Trinci ${ }^{9}$. Il s'agit d'un cas exemplaire où les apports du chercheur d'archive et l'analyse de l'historien de l'art s'opposent puisque ce cycle, attribué à Gentile en vertu de certaines sources documentaires, ne peut, pour des raisons stylistiques et qualitatives, avoir été réalisé par la main du maître mais plutôt par l'un de ses collaborateurs ou disciples. Le débat entre les partisans de cette paternité et leurs opposants est toujours ouvert et montre comment la méthode d'attribution et donc la lecture stylistique sur laquelle se fonde la philologie historico-artistique est désormais pratiquée avec beaucoup de légèreté, dans la mesure où les fresques de Foligno sont jugées par certains comme pouvant être rapprochées de façon cohérente des peintures données à l'artiste avec certitude.

La lecture stylistique a eu un rôle important dans l'approche de Gentile, non seulement pour la répartition des œuvres connues, mais surtout pour la reconnaissance du contexte de formation. C'est dans ce domaine que les monographies ont produit un apport significatif et diversifié : de l'idée première, la plus évidente et motivée par l'esprit de clocher, d'un enracinement dans la terre natale à l'ombre d'Allegretto Nuzi et Francescuccio Ghissi, idée héritée des légères monographies d'Arduino Colasanti (1909) et Bruno Molajoli (1927) ${ }^{10}$, à la thèse radicale de Luigi Grassi (à partir de l'examen du retable de Berlin; fig. 1) d'un lien de Gentile avec le milieu de l'enluminure lombarde de la fin du Trecento et en particulier avec les enlumineurs des tacuina sanitatis, Michelino da Besozzo et le "pointillisme " de Giovannino de Grassi ${ }^{11}$. Cette thèse a été ensuite soutenue par Bellosi ${ }^{12}$ puis De Marchi qui en développe la démonstration ${ }^{13}$, pendant qu'un avis différent privilégiant l'idée d'une formation vénitienne au début du Quattrocento au contact de Zanino di Pietro a été mise en avant par Christiansen : ce dernier s'est cependant montré favorable à cette piste de l'expérience lombarde dans la récente exposition de Fabriano ${ }^{14}$. Pour justifier historiquement de tels échanges, De Marchi s'appuie sur plusieurs éléments permettant

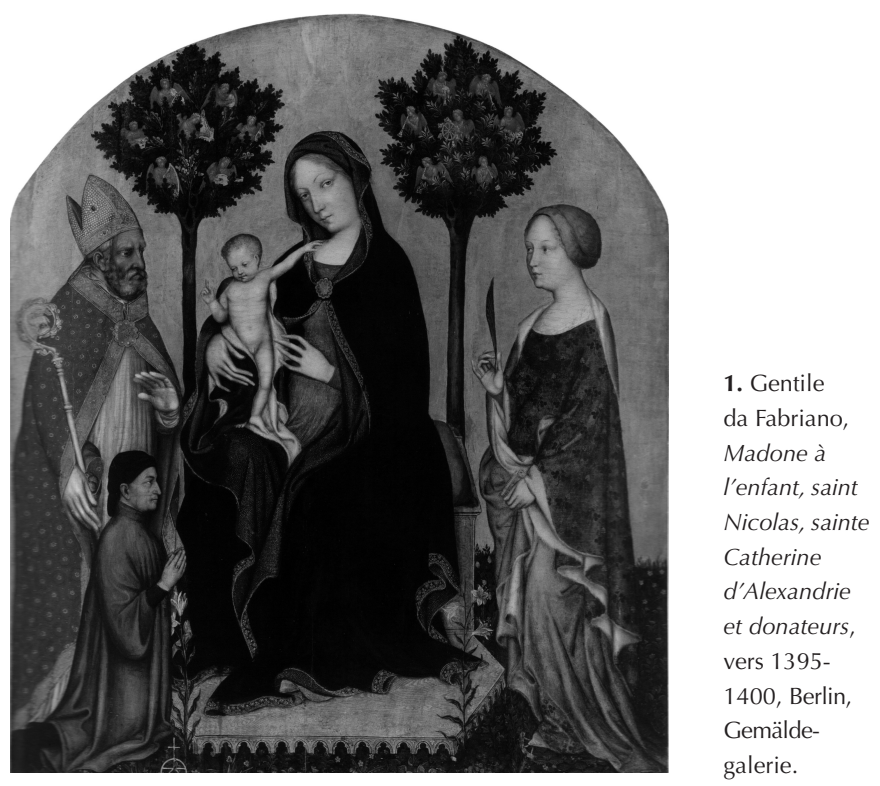


2. Gentile da Fabriano, Polyptyque Quaratesi (détail montrant saint Bernard de Clairvaux), 1425, Florence, Galerie des Offices.
3. Gentile da Fabriano, Polyptyque de I'Intercession, partie centrale (résultat des analyses avant la restauration), Florence, église San Nicolò Oltrarno. de supposer un voyage précoce de l'artiste à Pavie, notamment les contacts entre les Chiavelli, seigneurs de Fabriano, Antonio da Montefeltro et Gian Galeazzo Visconti ; de même les déplacements successifs de l'artiste sont mis en relation avec la disparition inattendue du duc de Milan en 1402, ou encore avec le trajet parcouru par Matin V à l'occasion de son lent rapprochement vers Rome dans le but de reconquérir le trône pontifical.

\section{Commandes et iconographie}

Les problèmes inhérents à la commande ont également été pris en compte dans le récent débat sur Gentile étranger à la question de la monographie. Il est désormais certain que le Polyptyque de l'Intercession de l'église San Nicolò Oltrarno à Florence (fig. 3) ne fut pas financé par la famille Quaratesi qui voulut en revanche le polyptique plus célèbre dispersé aujourd'hui entre différents musées (fig. 2). On le suppose originairement destiné à l'église de San Salvatore in Monte ${ }^{15}$, mais aussi, avec une argumentation fascinante, une commande de la part des Banchi, liés aux Strozzi et aux Quaratesi, et qui possédaient une chapelle à San Nicolò ${ }^{16}$. Dans le retable de Berlin (fig. 1), on a pensé, à partir de la marque placée à côté du dévot agenouillé, pouvoir reconnaître Ambrogio di Bonaventura, marchand de papier de Fabriano ${ }^{17}$, et la découverte d'un dessin du XVII ${ }^{\mathrm{e}}$ siècle dans la bibliothèque Mozzi Borgetti di Macerata semble lever les doutes quant à l'origine de l'œuvre ${ }^{18}$. En copiant le retable, l'auteur de ce dessin inscrivit sa provenance au revers de la feuille en indiquant l'église de San Nicolò di Fabriano, alors que dernièrement on avançait celle de Santa Caterina de Castelvecchio qui jouxte le couvent olivétain où le père de Gentile s'était retiré après 1385. Cette information n'a pas pour origine une étude sur Gentile ; elle est, pour cette raison, symptomatique de la façon dont certaines découvertes peuvent faire émerger des perspectives de recherche et produire de nouvelles pistes en apparence éloignées. En effet, dans cet exemple, il s'agissait de travaux me-

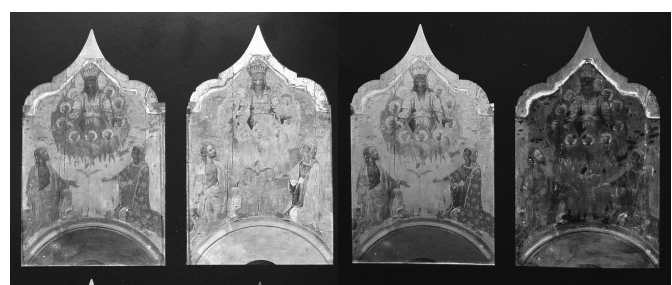

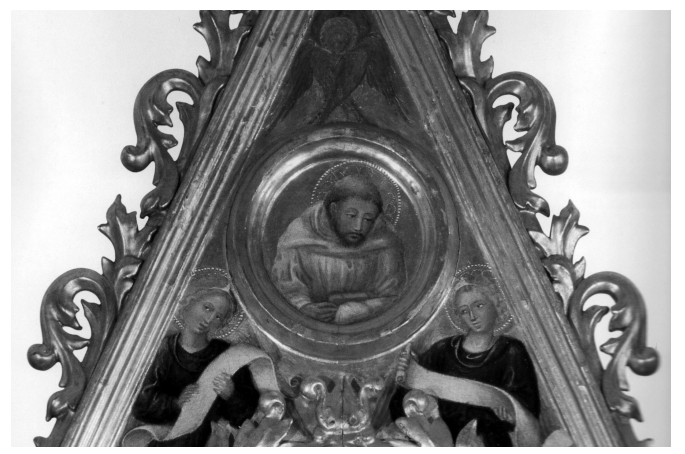

nés sur l'érudition historique et artistique dans les Marches entre les XVIII ${ }^{\mathrm{e}}$ et $\mathrm{XIX}^{\mathrm{e}}$ siècles, un terrain sur lequel de nombreuses recherches ont été effectuées ces dernières années et qui est probablement destiné à produire rapidement de fructueux résultats. La lecture d'une inscription jusque-là sousévaluée - au dos d'un des deux petits panneaux de la collection Berenson à Settignano (Florence) - a été le point de départ d'une reconstruction partielle du retable exécuté par Gentile pour la famille Sandei à Venise et destinée à l'église Sainte-Sophie ${ }^{19}$. Sans note et sans appareil critique approfondi, la monographie rédigée par Fabio Marcelli en 2005 est une compilation de thèses existantes mais elle contient cependant d'intéressantes observations sur la commande de la Madone de Pérouse tournant autour de l'élection de Bartolomeo degli Acerbi en 1404, prieur du couvent de San Domenico dans le prieuré de la province romaine de l'ordre des Frères Prêcheurs ${ }^{20}$.

L'analyse de l'iconographie est généralement considérée comme étant pertinente lorsqu'elle traite de problèmes strictement figuratif ou culturel, indépendamment des questions relatives à la provenance des œuvres. Mais, dans le cas de Gentile, certaines remarques ont fait la différence. Dans le cas du polyptyque Quaratesi anciennement à San Nicolò, la proposition selon laquelle il ne s'agissait pas de l'œuvre mentionnée dans les dispositions testamentaires de Bernardo di Castello Quaratesi en 1422 s'appuie, parmi différentes raisons invoquées, sur la volonté précise du commanditaire de voir représenté saint Bernard, son homonyme, qui ne figure manifestement pas dans l'œuvre alors qu'il apparaît dans le Polyptyque de l'Intercession qui se trouve dans la même église et qui fut pour cela considéré par quelques chercheurs comme l'œuvre commandée par Quaratesi pour 
l'autel principal, là où Vasari voyait l'œuvre la plus célèbre ${ }^{21}$. Parmi les arguments définitifs énoncés sur l'origine des deux polyptyques, Miklós Boskovits propose de reconnaître saint Bernard dans le buste du moine peint dans la flèche du retable conservé aujourd'hui aux Offices (Polyptyque Saint Nicolas; fig. 2), un détail généralement identifié comme un saint François mais qui présente les mêmes attributs iconographiques (tunique blanche, barbe et livre) que le saint homonyme peint dans le Polyptyque de l'Intercession ${ }^{22}$. Dans le livre de De Marchi aussi, on trouve des précisions iconographiques qui placent la conception de certains retables dans des contextes précis : l'iconographie expérimentale du " trône végétal " comprise comme le développement et l'exaltation du motif de l'hortus conclusus qui caractérise la Madone de Pérouse et fournit l'indice d'une exécution réalisée dans le milieu vénitien où l'on trouve d'autres exemples de cette invention originale ${ }^{23}$.

\section{Champs nouveaux I : les techniques}

Concernant Gentile, certains champs de recherches sont très nouveaux et par conséquent absents des monographies, comme celui du collectionnisme au XIX ${ }^{\mathrm{e}}$ siècle qui est apparu à l'occasion de l'exposition de Fabriano et ouvre une perspective critique sur le maître ${ }^{24}$.

L'étude systématique des différentes techniques élaborées par Gentile avec un art sans égal dans la peinture gothique tardive en Italie trouve son point de départ dans la monographie de De Marchi et a bénéficié des découvertes faites lors des analyses scientifiques des œuvres de Berlin (fig. 1) et Milan et, de manière plus approfondie, au cours des restaurations des polyptyques Quaratesi (fig. 2) et San Nicolò à Florence (fig. 3) et du retable Strozzi (Florence, Offices), sommet des expérimentations menées par le maître ${ }^{25}$. Ces travaux ont eu immédiatement des conséquences d'ordre culturel. En effet, le volume de De Marchi est axé sur les problèmes de réception technique des nouveautés de Gentile, en premier lieu le grainetis de l'or qui permet de simuler des figures évanescentes et légères. Dans une telle optique, il nous reste à identifier les personnalités directement influencées par le peintre ou fascinées par des techniques caractéristiques probablement dues à une connaissance indirecte du travail du maître, comme les panneaux de Nicola di Guardiagrele et du Pseudo Stefano di Ferrara que l'on a pu voir à l'exposition de Fabriano ; leur style est en effet indépendant des œuvres de Gentile.

\section{Champs nouveaux II : Gentile en contexte}

La reconstruction du contexte est au centre des monographies les plus importantes. L'ouvrage de Pietro Zampetti et Giampiero Donnini publié en 1992 est une synthèse présentée sous forme de répertoire, quelquefois imprécis, de la peinture à Fabriano entre la seconde moitié du XIII ${ }^{\mathrm{e}}$ siècle et la fin du $\mathrm{Xv}^{\mathrm{e}}$ siècle ${ }^{26}$. Gentile da Fabriano est principalement considéré dans le cadre des événements artistiques de sa ville d'origine, ses rapports avec les peintres de la région sont développés de façon générique. Le livre de Christiansen, au style concis et efficace, affronte immédiatement de telles questions, tantôt avec un ample point de vue, notamment lorsqu'il met en relation les recherches sur la lumière poursuivies par Gentile avec celles du Maître de Flémalle (Robert Campin) et celles du Maître des Heures de Boucicaut, tantôt avec une vue rapprochée, à propos du passage concernant le séjour du peintre à Florence. De cette façon, les échanges dialectiques sont mis en valeur, tout comme les différences de conception entre les sculpteurs et les peintres ayant opéré à cette époque ; le rôle de la fresque de la Piazza del Campo à Sienne aujourd'hui perdue est rapproché des œuvres subsistantes de Giovanni di Paolo et de Sassetta. Plus récemment, en 2005, le chercheur a formulé d'ambitieuses propositions sur le dialogue établi entre Gentile et les intellectuels florentins de l'époque; ses solides réflexions semblent confirmer que la définition d' "artiste gothique " est trop restrictive pour le maître de Fabriano ${ }^{27}$. Cet argument se précise lorsque l'on médite sur la portée figurative de la Pala Strozzi de 1423 réalisée lorsque Masaccio n'avait pas encore exprimé ses idées les plus originales : la prédelle (fig. 4) « demeure [...] la peinture la plus remarquable et la plus précieuse qui fut exécutée à ce moment-là au $\mathrm{XV}^{\mathrm{e}}$ siècle " ${ }^{28}$; " il est un fait que l'Adoration des Mages est une œuvre révolutionnaire, non pas à

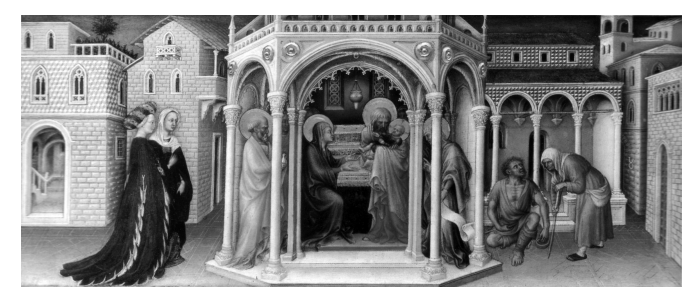

4. Gentile da Fabriano, Jésus présenté au temple (prédelle de la Pala Strozzi), 1423, Paris, Musée du Louvre. 
cause de la place sociale acquise par cet artiste dans la société ou pour sa relation avec le goût supposé de Palla Strozzi, mais parce qu'il exprime une nouvelle relation entre peinture et expérience $"{ }^{29}$, tendue vers l'exaltation du monde des apparences. La limite à franchir pour considérer Gentile comme incarnant une "autre Renaissance " ${ }^{30}$ n'est pas loin. L'ouvrage de De Marchi témoigne d'un parfait aboutissement dans la conception d'une monographie attentive aux réactions plus ou moins enthousiastes des différents contextes dans lequel évolua l'artiste, rythmée par des chapitres qui correspondent chacun à un centre important de cet itinéraire artistique. Avec les approfondissements consacrés à la réception stylistique du langage de Gentile, une ombre portée se dessine, distincte mais réelle, sur les vicissitudes de la peinture padouane du Quattrocento audelà des limites du gothique tardif et atteignant le jeune Vincenzo Foppa, pendant qu'en Toscane elle sera arrêtée presque immédiatement par un astre naissant, Masaccio, mais enveloppera cependant certains peintres sensibles aux vérités du monde phénoménologique, en premier lieu Fra Angelico.

\section{Les apports d'une exposition}

Cette vision aux multiples aspects, fondée sur la perméabilité de l'art de Gentile et sur les potentialités d'attraction stylistique et technique de ses peintures, a été offerte au visiteur de l'exposition qui, en 2006, s'est tenue dans la ville natale du peintre $^{31}$. Le parcours de la visite suivait les étapes du voyage de Gentile depuis sa formation, à la cour de Gian Galeazzo Visconti à Pavie, jusqu'à l'ambitieux chantier de Saint-Jean-deLatran à Rome qui marque la fin de sa carrière. Présentée ainsi, la conception de cette exposition monographique sans précédent

5. Orfèvre lombard et Maître du livre d'heures de Modène, Baiser de paix, fin XIVe- début Xve siècle, collection privée.

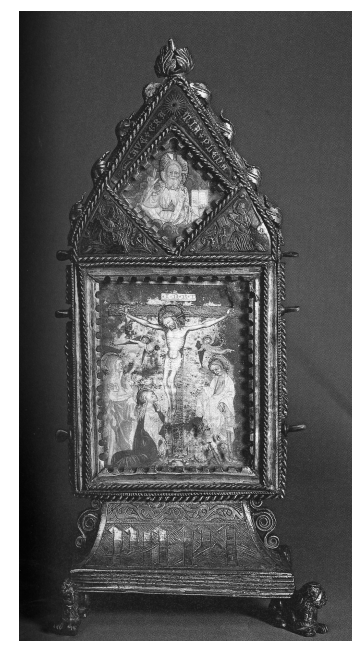

Gentile au sein de la production des personnalités actives dans les mêmes milieux figuratifs. Le rapprochement entre les œuvres a permis de mesurer concrètement comment certains artistes ont réagi à de telles stimulations en les interprétant différemment et parfois de façon très originale, depuis Arcangelo di Cola da Camerino jusqu'à Pisanello. Ainsi, on a pu voir comment le problème de la réception des modèles peut se limiter à la simple copie, comme les panneaux de Bicci di Lorenzo qui ne sont qu'une imitation de la prédelle du polyptyque Quaratesi. L'exposition présentait également des œuvres illustrant des conceptions figuratives parallèles et autonomes d'une aussi grande qualité que les compositions de Gentile lui-même : Michelino da Besozzo, Lorenzo Salimbeni, Masaccio. Les œuvres extraordinaires de Giovannino de Grassi et de Sassetta ouvraient et fermaient l'exposition et posaient respectivement les termes en rapport avec le travail de Gentile : un précédent et une réflexion sur le langage du maître.

Certaines attributions ont été proposées, comme le du dessin du Louvre avec le portrait de Gian Galeazzo Visconti ou l'Imago Pietatis de Prague; le problème de l'identification d'autres dessins attribués tantôt au maître, tantôt à ses disciples reste cependant ouvert. Parmi les objets les plus surprenants, il faut signaler la présence de chefs-d'œuvre d'orfèvrerie transalpine, en particulier française et germanique, qui ont mis en évidence l'origine de certaines spécificités techniques adoptées par Gentile dès ses premières ouvres et en particulier le grainetis de l'or : la démonstration en était faite par la Croix reliquaire de Solingen-Gräfrath et le triptyque de cuivre et d'argent du Metropolitan Museum de New York présentés aux côtés de pièces d'orfèvreries contenant des miniatures, comme l'œuvre d'un orfèvre lombard qui collabora avec le Maître du Livre d'Heures de Modène (fig. 5). Ce qui a soutenu cette ambition de construire un réseau intense de dialogues, correspondances, dérivations ou au contraire le choix d'autonomie, est la sélection rigoureuse des cas examinés et donc des œuvres exposées. La seule exception était une section de l'exposition consacrée à la peinture produite à Fabriano au Quattrocento, quelque peu prolixe dans son commentaire des itinéraires des artistes liés à Gentile comme le Maestro di Staffolo et Antonio da Fabriano, mais la raison de sa présence s'expliquait par le fait qu'elle faisait honneur à la ville qui accueillait l'exposition. 
Le catalogue de l'exposition et le volume d'essais qui le complétait apportaient approfondissements et mises à jour sur les informations que nous possédons sur Gentile. Les nouveautés importantes concernaient essentiellement les peintres influencés par lui, comme Pellegrino di Giovanni à propos duquel de nouvelles sources ont été découvertes ${ }^{32}$, et des problématiques particulières, comme la reconstruction du polyptyque vénitien de Sainte-Sophie $^{33}$. Dans ce contexte de recherche, l'évaluation de la production de l'atelier qui comprend des personnalités anonymes ou dont l'identité n'est que supposée a permis de constituer la géographie de Gentile qui s'étend de la Vénétie à l'Ombrie ${ }^{34}$. Cet état de fait est naturellement dû aux déplacements et à la diffusion des ouvres du maître que nous connaissons depuis Vasari qui déclarait avec une emphase, probablement due au désir d'exalter le rôle de Gentile en Italie centrale, qu' « il travailla beaucoup dans les Marches, notamment à Gubbio où certaines de ses œuvres sont toujours visibles, dans la région d'Urbin ${ }^{35}$.

Dans l'ensemble, les travaux que nous venons de commenter montrent qu'il n'est pas nécessaire de mettre au jour des œuvres inédites du peintre pour construire une monographie solide. Celle de Christiansen, élaborée à partir d'un corpus très sélectionné, n'en apporte aucune, tout comme l'exposition de Fabriano; l'autre, de De Marchi, est enrichie par la découverte des fragments du cycle de Brescia, de la Crucifixion conservée aujourd'hui à la Pinacoteca di Brera et de quelques nouvelles propositions d'attribution qui ne représentent cependant pas l'apport le plus important de l'ouvrage. D'autres découvertes fortuites comme les fragments d'une frise végétale à Saint-Jean-de-Latran à Rome ${ }^{36}$ et les deux petits panneaux de la Pinacoteca Nazionale di Bologna ${ }^{37}$ sont des éléments récents.

$\mathrm{Au}$ regard des résultats trouvés, les pistes de recherche examinées rapidement dans cet article pourraient être approfondies, mais pour l'heure, il semble qu'une nouvelle monographie sur Gentile da Fabriano ne soit pas des plus opportunes.

1. Andrea De Marchi, Gentile da Fabriano. Un viaggio nella pittura italiana alla fine del gotico, Milan, 1992, p. 7. Il existe une réimpression récente de ce volume non mis à jour mais avec un nouvel avant-propos (Milan, 2006).
2. Luigi Grassi, Tutta la pittura di Gentile da Fabriano, Milan, 1953.

3. Keith Christiansen, Gentile da Fabriano, Ithaca (N.Y.), 1982 ; De Marchi, 1992, cité n. 1. La structure de l'ouvrage d'Emma Micheletti est issue de la monographie de Grassi : Emma Micheletti, L'opera completa di Gentile da Fabriano, Milan, 1976

4. Adolfo Venturi, Le vite de' più eccellenti pittori, scultori e architettori scritte da m. Giorgio Vasari pittore et architetto aretino. I. Gentile da Fabriano e il Pisanello. Edizione critica con note, documenti e 96 riproduzioni, Florence, 1896.

5. Pour une synthèse et aussi pour les informations qui précèdent, je renvoie le lecteur à la documentation complète sur l'artiste rédigée à l'occasion de l'exposition de Fabriano en 2006 : Matteo Mazzalupi, "Regesto », dans Andrea De Marchi, Laura Laureati, Lorenza Mochi Onori éd., Gentile da Fabriano. Studi e ricerche, Milan, 2006, p. 68-84.

6. Bartolomeo Facio, De viris illustribus (vers 1453-1457), cité d'après Michael Baxandall, Les humanistes à la découverte de la composition en peinture. 1340-1450, Paris, 1971 (1989), p. 132-133.

7. Giorgio Vasari, Le vite dé più eccelenti architetti, pittori e scultori italiani, da Cimabue Insino a' tempi nostri, Florence, 1568, parte IV.

8. Stefano Felicetti, "Regesti documentari (1299-1499)", dans Fabio Marcelli éd., Il Maestro di Campodonico. Rapporti Artistici fra Umbria e Marche nel Trecento, Fabriano, 1998, p. 217, doc. 93-94 ; Fabio Marcelli, Gentile da Fabriano, Milan, 2005. p. 32 .

9. Voir les contributions réunies dans Giordana Benazzi, Francesco Federico Mancini éd., Il Palazzo Trinci di Foligno, Pérouse, 2001.

10. Arduino Colasanti, Gentile da Fabriano, Bergame, 1909, p. 32 ; Bruno Molajoli, Gentile da Fabriano, Fabriano, 1927, p. 22-23.

11. Grassi, 1953, cité n. 2, p. 7-12, 40, 50.

12. Luciano Bellosi, Gentile da Fabriano (I Maestri del Colore, 159), Milan, 1966, p. [4].

13. De Marchi, cité n. 1, p. 11-45.

14. Christiansen, cité n. 3; Keith Christiansen, "L'arte di Gentile da Fabriano ", dans Laura Laureati, Lorenza Mochi Onori éd., Gentile da Fabriano e l'altro Rinascimento (cat. expo., Fabriano, Spedale di Santa Maria del Buon Gesù, 2006), Milan, 2006, p. 25-28.

15. Cecilia Frosinini, "L'Intercessione. Il "nuovo" polittico di Gentile da Fabriano ritrovato ", dans Marco Ciatti, Cecilia Frosinini éd., Il "Polittico dell'Intercessione" di Gentile da Fabriano. Studi e restauro, Florence, 2006, p. 17-38.

16. Annamaria Bernacchioni, "Firenze ", dans De Marchi, Laureati, Mochi Onori, cité n. 5, p. 123-130.

17. Fabio Marcelli, "Presenze, committenza e dispersione : quattro schede sul patrimonio artistico di Pergola, Fabriano e Fermo ", dans Notizie da Palazzo Albani, XXII-XXIX, 19932000, p. 77-80. 
18. Anna Maria Ambrosini Massari, « Ricci, Maggiori, Gentile : la nascita della storia dell'arte nelle Marche e un disegno ", dans Cecilia Prete éd., Gentile da Fabriano, "Magister Magistrorum", (colloque, Fabriano, 2005), Senigallia, 2006, p. 129-146. L'œuvre ne fut en possession de la famille Ottoni de Matelica que dans un second temps : Alberto Bufali, "Nuovi documenti per Carlo Crivelli e Luca Signorelli ed una ipotesi per gli inizi di Gentile da Fabriano ", dans Pierluigi Moriconi éd., Storie da un archivio : frequentazioni, vicende e ricerche negli archivi camerinesi (colloque, Camerino, 2006), Camerino, 2006, p. 43-50 et 62.

19. Cette proposition a été développée à l'occasion de l'exposition de Fabriano : Matteo Ceriana, Emanuela Daffra, " Il Polittico di Valle Romita : la sua storia nel museo ", dans Matteo Ceriana, Emanuela Daffra éd., Gentile da Fabriano. Il Polittico di Valle Romita, (cat. expo., Milan, Pinacoteca di Brera, 1993), Milan, 1993, p. 27-30 ; Eadem, dans Laureati, Mochi Onori, 2006, cité n. 14, p. 140-143.

20. Marcelli, 2005, cité n. 8, p. 54.

21. Pour une synthèse sur ce sujet, voir Frosinini, 2006, cité n. 15 , p. 20-21.

22. Miklós Boskovits, dans Luciano Bellosi éd., Masaccio e le origini del Rinascimento, (cat. expo., San Giovanni Valdarno, Casa Masaccio, 2002), Milan, 2002, p. 160-163. Alessandro Cecchi, dans Laureati, Mochi Onori, 2006, cité n. 14, p. 256261, ignore cette contribution et affirme qu'il n'y a aucune trace de l'image de saint Bernard, mais, paradoxalement, l'intitulé de la notice précise qu'il s'agit de saint Nicolas de Bari et saint Bernard.

23. De Marchi, 1992, cité n. 1, p. 49-51.

24. Laura Laureati, "Gentile, scomparso, torna a Fabriano dopo qualche secolo. Una breve storia della dispersione ottocentesca di alcune opere di Gentile e della loro parziale ricomposizione in occasione della mostra ", dans Laureati, Mochi Onori, 2006, cité n. 14, p. 53-59 ; Elisabetta Federici, " Gentile da Fabriano nei taccuini di un connoisseur inglese : Charles Lock Eastlake ", dans De Marchi, Laureati, Mochi Onori, 2006, cité n. 5, p. 143-153. Sur les déplacements des œuvres de Gentile da Fabriano au $\mathrm{Xv}^{\mathrm{e}}$ siècle, le travail de Stefano L'Occaso est essentiel : Fonti archivistiche per le arti a Mantova tra Mediovo e Rinascimento (1382-1459), Mantoue, 2005, p. 216, 222.

25. Voir les essais de Nicola Ann MacGregor et Sandra Freschi, dans Alessandro Cecchi éd., Gentile da Fabriano agli Uffizi, Milan, 2005, p. 173-180 et p. 181-186; ceux du volume de Ciatti, Frosinini, 2006, cité n. 15 ; voir aussi Roberto Bellucci, Cecilia Frosinini, «Tecnica e stile : appunti su Gentile da Fabriano ", dans De Marchi, Laureati, Mochi Onori, 2006, cité n. 5, p. 55-65, et les contributions ajoutées au dossier de Ceriana, Daffra, cité n. 19.

26. Pietro Zampetti, Giampiero Donnini, Gentile e $i$ pittori di Fabriano, Florence, 1992.

27. Keith Christiansen, "L'Adorazione dei Magi di Gentile da Fabriano », dans Cecchi, 2005, cité n. 25, p. 11-40.

28. Christiansen, 1982, cité n. 3, p. 37 : «... remains [...] the most remarkable and precocious painting executed up to that moment in the fifteenth century ".
29. Ibidem, p. 64 : "The fact remains that the Adoration of the Magi is a revolutionary work not because of the social standing of its artist or its relation to the hypothetical taste of Palla Strozzi, but because it expounds a new relation between painting and experience ".

30. De Marchi, 1992, cité n. 1, p. 9.

31. Laureati, Mochi Onori, 2006, cité n. 14; De Marchi, Laureati, Mochi Onori, 2006, cité n. 5.

32. Maria Rita Silvestrelli, dans Laureati, Mochi Onori, 2006, cité n. 14, p. 118-120.

33. Voir note 19.

34. Andrea De Marchi, "Gentile e la sua bottega ", dans De Marchi, Laureati, Mochi Onori, 2006, cité n. 5, p. 9-53.

35. Vasari, 1568, cité n. 7, p. 51.

36. Andrea De Marchi, " Gentile da Fabriano et Pisanello à Saint-Jean de Latran ", dans Dominique Cordellier, Bernadette Py éd., Pisanello, (colloque, Paris, 1996), Paris, 1998, vol. 1, p. 161-213.

37. Daniele Benati, dans Jadranka Bentini, Gian Piero Cammarota, Daniela Scaglietti Kelescian éd., Pinacoteca Nazionale di Bologna. Catalogo generale. 1. Dal Duecento a Francesco Francia, Venezia, 2004, p. 180-181.

Mauro Minardi, Università degli Studi della Basilicata

mauromi@katamail.com

\section{Carlo Crivelli : style ou iconographie Thomas Golsenne}

La bibliographie du peintre d'origine vénitienne Carlo Crivelli (v. 1435 - v. 1494) commence par une lacune. Crivelli fait en effet partie des rares grands artistes de la Renaissance absents des Vite de Vasari. Les raisons de cette absence sont aisées à imaginer. La Marche d'Ancône, où travaillait Crivelli, ne comptait pas à l'époque de Vasari de grands centres artistiques, et l'art toscan - le meilleur, selon Vasari - avait peu pénétré dans le sud de la région, où Crivelli opérait. En outre, le style même de Crivelli devait susciter peu d'intérêt de la part du biographe arétin : s'il avait regardé ses polyptyques, illes avait probablement considérés commelesœuvres d'un lointain disciple de Gentile da Fabriano, trop gothiques, trop décoratifs ; autrement dit, d'un peintre qui n'avait pas su prendre 